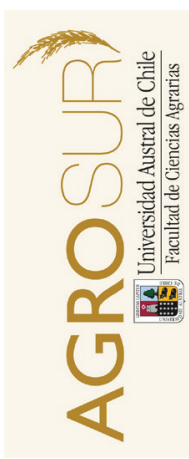

\title{
Efecto del estrés hidráulico y mecánico sobre la resistencia y resiliencia funcional del sistema poroso de un Ñadi (Aquands) bajo distintos usos de suelo
}

\author{
Effect of hydraulic and mechanical stresses on the functional resistance and \\ resilience of the porous system of a Ñadi (Aquands) under different land uses.
}

\author{
Haller, P. ${ }^{a *}$, Dec, D. ${ }^{a, b}$, Zúñiga, F. ${ }^{a, b, c}$, Thiers, $\mathbf{0}^{b, d}$, Ivelic-Sáez, J. ${ }^{e}$, Horn, R. ${ }^{b, f}$, Dörner, J. ${ }^{a, b *}$ \\ ${ }^{a}$ Instituto de Ingeniería Agraria y Suelos. Facultad de Ciencias Agrarias, Universidad Austral de Chile, Valdivia, Chile. \\ ${ }^{b}$ Centro de Investigación en Suelos Volcánicos, Universidad Austral de Chile, Valdivia, Chile. \\ 'Programa de Doctorado en Ciencias Agrarias, Escuela de Graduados, Facultad de Ciencias Agrarias, \\ Universidad Austral de Chile, Valdivia, Chile.
}

${ }^{d}$ Instituto de Bosque y Sociedad. Facultad de Ciencias Forestales y Recursos Naturales, Universidad Austral de Chile, Valdivia, Chile.

e्enstituto de Investigaciones Agropecuarias, INIA-Kampenaike. Angamos N1056, Punta Arenas, Chile.

fInstitut für Pflanzenernährung und Bodenkunde, Christian Albrechts Universität zu Kiel Olshausenstrasse 40, 24118 Kiel, Germany.
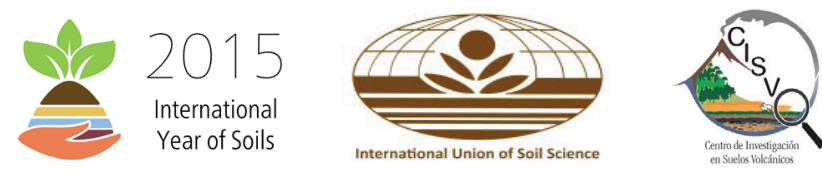

\begin{tabular}{l} 
A R T I C L E I N F O \\
\hline Article history: \\
Received 31.07.2015 \\
Accepted 22.12.2015 \\
\hline Keywords: \\
Mechanical stability \\
Pore functionality \\
History of land use \\
COLE \\
\hline Original Research Article, \\
Special Edition: International \\
Year of Soils (IYS) \\
Soil Science \\
\hline *Corresponding author: \\
Pía Haller, José Dörner \\
E-mail address: \\
piahallerc@gmail.com, \\
josedorner@uach.cl
\end{tabular}

The aim of the study was to analyze the functional resistance and resilience of the pore system of a Ñadi soil subjected to mechanical and hydraulic stresses. Undisturbed soil samples were collected at two depths (5 and $15 \mathrm{~cm}$ ) in a Duric histic placaquands (series Alerce) under different land uses (natural forest: BN - naturalized grassland: PN). Air capacity (CA), plant available water (ADP) and air conductivity $\left(k_{l}\right)$ were determined before and after the soil had been subjected (separately) to hydraulic (in pressure cooker) and mechanical (in odometer) stress. The coefficient of linear extensibility (COLE) was used to quantify the resistance and resilience. The land use change caused a decrease of soil depth, exposing the A horizon in the soil surface under PN. The soil under BN was less resistant due to its higher COLE after being subjected to both applied stresses, however, it showed the higher mechanical resilience. The soil deformation at $5 \mathrm{~cm}$ in BN induced a decrease of CA and $k_{l}$ as well as a higher volume of ADP. The hydraulic and mechanical history of the soil under the naturalized pasture (PN), increased the mechanical stability which allows to maintain soil pore functions. The functional resistance and resilience of soil pores determined by COLE correlate positively with the organic carbon content (CO).

josedorner@uach.cl

\section{RESUMEN}

El objetivo del presente trabajo fue analizar el efecto del uso actual de un suelo Ñadi sobre la resistencia y resiliencia funcional de sus poros, luego de someter al suelo a eventos de estrés mecánico e hidráulico. Se recolectaron muestras no disturbadas de suelo a dos profundidades ( 5 y $15 \mathrm{~cm}$ ) en un Duric histic placaquands (serie Alerce) bajo dos usos de suelo (bosque nativo: BN pradera naturalizada: PN). Se determinó la capacidad de aire (CA), el agua disponible para las plantas (ADP) y la conductividad de aire $\left(k_{l}\right)$ antes y después de someter las muestras a un estrés hidráulico (en ollas de presión) y mecánico (en un odómetro), por separado. Se determinó el coeficiente de extensibilidad lineal (COLE) para cuantificar la resistencia y resiliencia. El cambio de uso de suelo provocó una reducción de la profundidad y la exposición del horizonte A en la superficie del suelo bajo PN. El suelo bajo BN, fue menos resistente ya que presentó el mayor COLE luego de ambos tipos de estrés, sin embargo, presentó la mayor resiliencia. La deformación del suelo a los $5 \mathrm{~cm}$ en BN significó una reducción de la CA y $k_{l}$ pero un incremento en los poros de ADP. El historial hídrico y mecánico del suelo bajo PN, le confiere una mayor estabilidad, manteniendo el funcionamiento del sistema poroso. La resistencia y resiliencia funcional de los poros del suelo determinada mediante el COLE se correlacionan de forma positiva con el contenido de carbono orgánico (CO).

Palabras clave: Estabilidad mecánica, funcionamiento de los poros, historial de uso del suelo, COLE. 


\section{INTRODUCCIÓN}

Para sustentar la demanda alimentaria mundial es fundamental tener suelos de calidad. En este contexto, Karlen et al. (1997), proponen definir la calidad de suelo como "la capacidad de un tipo específico de suelo de funcionar, dentro de límites ecosistémicos naturales o manejados, para sostener la productividad vegetal y animal, mantener o aumentar la calidad del aire y agua y sostener la salud y hábitat humano". Los suelos que presentan una buena calidad física (p.ej. $20 \%$ de volumen de agua aprovechable para las plantas, Reynolds et al., 2009; > 8\% de capacidad de aire, Horn y Fleige, 2009) permiten promover al máximo el desarrollo de los cultivos y minimizar la degradación ambiental (Ferreras et al., 2007). Bajo este prisma la calidad física del suelo puede evaluarse a través de la resistencia y resiliencia de los poros del suelo. Mientras el primero corresponde a la capacidad de un suelo de mantener sus funciones sin variaciones significativas luego de ser expuesto a un estrés, la resiliencia se define como la habilidad del suelo para recuperarse después de un estrés (Seybold et al., 1999; Dörner et al., 2011). La resiliencia es un término que apunta a la sustentabilidad del recurso suelo, con el fin de combatir la degradación de éste, lo que además nos permite analizar la habilidad que tienen un tipo específico de suelo de recuperar su integridad estructural y funcional después de realizada una perturbación (Dörner et al., 2009).

Para lograr un aumento en la superficie de tierras arables, hace 300 años que los humanos han transformado gran parte de la superficie planetaria, llevando consigo un cambio drástico del paisaje mediante la tala de bosques, el incremento de la intensificación de la producción agrícola y la expansión de los centros urbanos (Aguayo et al., 2009). En ese contexto, para incrementar la superficie de suelo para la agricultura, en la zona sur de Chile se ha producido un importante cambio de uso de suelo en los últimos 200 años (Martínez et al., 2008). Entre esos suelos se encuentran aquellos denominados Ñadi (Placaquands), los que derivan de cenizas volcánicas de tipo andesítico basálticas depositadas sobre un sustrato fluvioglacial (Luzio, 2010). Thiers et al. (2007) mencionan que la principal característica de este tipo de suelo es la presencia de un anegamiento temporal en períodos de máxima precipitación, debido -en parte- a la presencia de un horizonte plácico, que corresponde a un horizonte cementado o endurecido por hierro y manganeso (Soil Survey Staff, 2006). La presencia del horizonte plácico hace que el drenaje interno del suelo sea muy lento, produciendo una saturación total del perfil de suelo, la cual puede durar entre cuatro a seis meses, dificultando su manejo y limitando su uso (Thiers et al., 2007). Por lo tanto, son suelos de características extremas, ligeramente profundos (40 - 70cm, según SAG, 2001) y con limita- ciones tanto físicas como químicas, lo que impide su uso para la producción agrícola o ganadera sin un previo tratamiento de estas limitaciones.

Los suelos están expuestos a los estreses del medio y a los generados por su uso. Mientras el pisoteo animal y el tránsito de maquinaria agrícola generan estreses de tipo mecánico, los ciclos de mojado y secado del suelo inducen un estrés de tipo hidráulico. Ambos procesos, se caracterizan por ser cíclicos y tener diferentes efectos sobre el funcionamiento de los poros del suelo (Dörner et al., 2011). En ese contexto, el estrés hidráulico puede generar cambios beneficiosos para la estructura del suelo (Ivelic-Sáez et al., 2015), en comparación al estrés mecánico que provoca un deterioro de la estructura (Dörner et al., 2009). Estos tipos de estreses se pueden acentuar con el cambio de uso de suelo (Dörner et al., 2011), por lo tanto, evaluar el efecto de ambos tipos de estrés bajo las condiciones particulares de formación y uso de los suelos Ñadi es muy relevante, debido a que poseen una limitada profundidad total y alta porosidad, por lo que están potencialmente expuestos a una deformación irreversible, lo que hasta la fecha no ha sido investigado en el sur de Chile. Por lo tanto, el objetivo de este estudio, es analizar el efecto del uso actual del suelo sobre la resistencia y resiliencia funcional de sus poros, sometidos a eventos de estrés mecánico e hidráulico, en una condición de suelo Ñadi (Aquands) en la región de Los Lagos, Chile.

\section{MATERIALES Y MÉTODO}

\section{Sitio e historial de manejo}

Esta investigación se llevó a cabo en el predio Chaqueihua, ubicado en el sector Tepual, a 17 kilómetros al

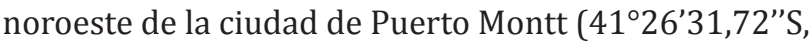
737'51,70"0; 70-74 msnm). La unidad de investigación consta actualmente de dos usos de suelo: bosque nativo (BN) y pradera naturalizada (PN). El BN corresponde al tipo forestal siempre verde y dominan las especies: Nothofagus dombeyi (Mirb.) Oerst., Drimys winteri J.R.Forst. \& G.Forst., Podocarpus nubigena Lindl., y Amomyrtus luma (Molina) D.Legrand \& Kausel. La PN, contigua al bosque nativo, deriva de un incendio accidental ocurrido hace 33 años en el sector. Terminado el siniestro, se quitaron los restos de tocones presentes, habilitando la pradera naturalizada, la cual no registra eventos de mejoramiento (fertilizaciones o enmiendas calcáreas), labranza de suelo, ni manejos del pastoreo desde su formación. Actualmente, la pradera está constituida principalmente por: Aira Caryophyllea L., Holcus lanatus L. y Agrostis capillaris L.

El clima es del tipo templado oceánico o lluvioso, con ausencia de período seco, ya que las precipitaciones se distribuyen a lo largo de todo el año, la precipitación promedio para esta zona varía de 1.800 a $2.500 \mathrm{~mm}$ 
anuales y se caracteriza por tener una temperatura promedio anual de $11,2^{\circ} \mathrm{C}$ (Sánchez y Morales, 2000). Las características edáficas y los regímenes de humedad ácuicos clasifican a este suelo como un Duric Histic Placaquands, que se denomina localmente como serie Alerce. Este tipo particular de Andisol ocupa posiciones planas y deprimidas del paisaje, teniendo un drenaje imperfecto por largos periodos al año. Son suelos ligeramente profundos $(40-70 \mathrm{~cm})$, que se desarrollaron a partir de cenizas volcánicas recientes depositadas sobre un sustrato fluvioglacial. Entre el suelo y el sustrato existe una capa denominada "Fierrillo", con un espesor de hasta $4 \mathrm{~mm}$, irregular, discontinuo y de cementación variable, generando condiciones de saturación que se modifican durante el año. El sustrato fluvioglacial constituye frecuentemente un duripán, por la fuerte cementación silícica presente. También, existe presencia de un horizonte 0 (orgánico), sobre el suelo mineral, de hasta $10 \mathrm{~cm}$ de espesor (CIREN, 2003).

\section{Muestreo de suelo}

Se utilizó un bastón agrológico para determinar la homogeneidad del suelo en función de su profundidad efectiva y variabilidad morfológica. Luego se realizó una calicata, en donde se llevó a cabo la caracterización del suelo, definiendo el espesor de sus horizontes, límites y propiedades morfológicas para cada uso de suelo.

En cilindros metálicos de $230 \mathrm{~cm}^{3}$ (diámetro $=5,73$; altura $=7,15 \mathrm{~cm}$ ) se recolectaron muestras de suelo no disturbado en cada uso: BN y PN a 5 y $15 \mathrm{~cm}$ de profundidad en invierno del 2013, para determinar la curva de retención de agua y la distribución de poros. La recolección de muestras se realizó en función de la profundidad del suelo tomando como referencia el horizonte superficial donde el impacto de los estreses sobre el suelo es más intenso. La totalidad de muestras consideradas para esta investigación fueron de 56 cilindros (28 cilindros por uso de suelo). Del total de muestras, se eliminaron cinco que presentaron valores de conductividad de aire fuera de rango (mayores que el doble de la desviación estándar, DS).

Adicionalmente, se colectó material para realizar una caracterización general del suelo. Para este tipo de material se llevaron a cabo los siguientes análisis: clase textural al tacto in situ (Schlatter et al., 2003), densidad real $\left(\rho_{s}\right)$ y densidad aparente $\left(\rho_{a}\right)$ según Forsythe (1985), aluminio extractable $\left(\mathrm{Al}_{\text {ext. }}\right)$ y carbono orgánico (CO) de acuerdo a Sadzawka et al. (2006).

\section{Análisis de laboratorio}

El experimento consistió en realizar una primera curva de retención de agua $\left(\mathrm{C}_{1}\right)$ equilibrando las muestras de suelo a $0,1,2,3,6,15,33,50 \mathrm{kPa}$ de tensión mediante ollas de presión (Dörner et al., 2013); en cada una de estas tensiones se registró el peso de las muestras con una balanza electrónica (Precisa 1000C-3000D, 0,01 de precisión) y la deformación unidimensional con un profundímetro electrónico (Vernier Caliper, 0,01 mm precisión). Además, desde 6 a $50 \mathrm{kPa}$, se midió la conductividad de aire $(k)$ con un flujómetro de aire (Dörner et al., 2009). Una vez finalizada $C_{1}$, las muestras se saturaron hasta lograr equilibrarlas nuevamente a $0 \mathrm{kPa}$ (se midió peso y contracción), posteriormente fueron llevadas, a $6 \mathrm{kPa}$ de tensión (en donde también se midió $k_{l}$ ), en esta condición se aplicaron ambos tipos de estrés: hidráulico y mecánico. Para el primer estrés (hidráulico) se aplicaron $80 \mathrm{kPa}$ de tensión (por medio de cámaras de presión) y para el estrés mecánico se aplicaron $80 \mathrm{kPa}$ de presión (usando un odómetro de pistón). Según Krümmelbein et al. (2006) ésta es la presión estática que produce un ovino sobre el suelo.

Para someter las muestras al estrés hidráulico estas estuvieron aproximadamente 14 días dentro de las ollas de presión, ya que se ha determinado para suelos volcánicos (Duric hapludand, serie Valdivia) que en este tiempo se logra alcanzar el equilibrio a $80 \mathrm{kPa}$ de presión atmosférica (Ivelic-Sáez et al., 2015). Posteriormente, se midió la contracción de la muestra, el contenido gravimétrico de agua y la conductividad de aire.

Durante el ensayo del estrés mecánico (mediante un odómetro), se registró la deformación vertical de cada muestra durante 6 minutos cada 30 segundos. Al retirar el estrés, se determinó la recuperación de cada muestra de suelo cada 30 segundos durante 6 minutos. El estrés mecánico tuvo una duración total de $12 \mathrm{mi}$ nutos. Al concluir el tiempo de recuperación se midió la deformación unidimensional del suelo y, posteriormente, el contenido de agua y $k_{r}$. Finalizada la aplicación de ambos tipos de estrés, las muestras se equilibraron nuevamente a $0 \mathrm{kPa}$ y se realizó una segunda curva de retención de agua $\left(\mathrm{C}_{2}\right)$, incluyendo las mismas variables de medición que en $\mathrm{C}_{1}$. En la Figura 1 se presenta el resumen general del experimento para el índice de poros $\left(\mathrm{IP}=\left[\rho_{s} / \rho_{a}\right]-1\right)$.

Para determinar el contenido de humedad de cada muestra, éstas se secaron a $105^{\circ} \mathrm{C}$ por 48 horas. Posteriormente, se midió la altura y peso de cada muestra con lo que se determinó el contenido volumétrico de agua y el índice de poros en cada tensión aplicada, corrigiéndola según la deformación vertical del suelo producto de la contracción (Dörner et al., 2009).

Las curvas de retención de agua y de contracción fueron utilizadas para definir parámetros de capacidad e intensidad (Horn y Kutilek, 2009). Como parámetros de capacidad se consideraron el volumen de agua disponible para las plantas (ADP) y la capacidad de aire (CA), los que derivan de la curva de retención de agua. Para definir parámetros de intensidad se utilizó la conductividad de aire $\left(k_{l}\right)$. Para evaluar la resistencia y resiliencia se utilizó la curva de contracción. A partir 


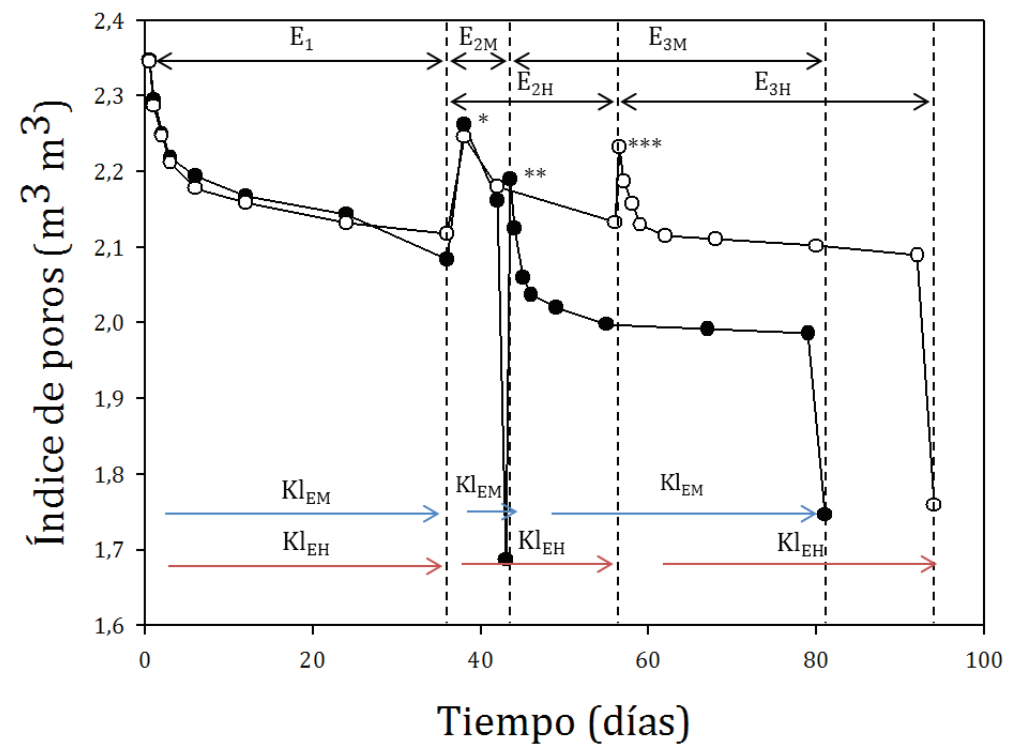

Donde:

$\mathrm{E}_{1}$ : primera curva de retención de agua, tanto para el estrés mecánico (círculos negros), como para estrés hidráulico (círculos blancos), $\mathrm{E}_{2 \mathrm{M}}$ : Inicio y término de estrés mecánico, $\mathrm{E}_{2 \mathrm{H}}$ : inicio y término del estrés hidráulico, $\mathrm{E}_{3 \mathrm{M}}$ : inicio y término de segunda curva de retención de agua del estrés mecánico, $\mathrm{E}_{3 \mathrm{H}}$ : inicio y término de segunda curva de retención de agua del estrés hidráulico. $\mathrm{Kl}_{\mathrm{EM}}$ : mediciones de la conductividad de aire para el estrés mecánico y $\mathrm{Kl}_{\mathrm{ЕН}}$ : mediciones de la conductividad de aire para el estrés hidráulico. (*): Punto indicando 6 kPa de tensión después de $\mathrm{E} 1$ en ambos estreses, $\left(^{* *}\right)$ : punto indicando $0 \mathrm{kPa}$ después de dos días de recuperación para estrés mecánico y (***): Punto indicando 0 kPa después de dos días de recuperación para estrés hidráulico.

Fuente: Adaptado de Ivelic (2013).

Figura 1. Esquema del experimento de laboratorio: evolución del índice de poros (IP) en el tiempo.

Figure 1. Chart representing the laboratory experiment: temporal evolution of void ratio (IP).

de dicha curva, se determinó el coeficiente de extensibilidad lineal (COLE) que define la deformación unidimensional del suelo desde un punto de saturación, a condiciones de menor contenido de agua (Dörner et al., 2009). El COLE, permite caracterizar la deformación y recuperación del suelo. Este último parámetro se midió antes y después para cada uno de los tipos de estrés. El COLE c se usó para determinar la resistencia y el COLE r permitió ver la recuperación (resiliencia) del suelo después de ambos tipos de estrés. Para ambos tipos de estrés se usaron las siguientes ecuaciones:

$$
\begin{aligned}
& \text { COLE c }=\left(\mathrm{L}_{80 \mathrm{kPa}}-\mathrm{L}_{0 \mathrm{kPa}}\right) /\left(\mathrm{L}_{80 \mathrm{kPa}}\right) \quad \text { Ec. } 1 \\
& \operatorname{COLE~} \mathrm{r}=\left(\mathrm{L}_{0 \mathrm{kPa}}-\mathrm{L}_{80 \mathrm{kPa}}\right) /\left(\mathrm{L}_{80 \mathrm{kPa}}\right) \quad \text { Ec. } 2
\end{aligned}
$$

Donde:

$\mathrm{L}_{0 \mathrm{kPa}}$ : altura de la muestra a $0 \mathrm{kPa}$ y $\mathrm{L}_{80 \mathrm{kPa}}$ : altura de la muestra a $80 \mathrm{kPa}$.

Según Peng et al. (2007) los índices del COLE pueden variar entre $<0,03$ y $>0,09$ y cuantifican la magnitud de la contracción o expansión del suelo. La resiliencia (COLE r) es una recuperación de la altura de la muestra posterior al efecto del estrés y es por ello que presenta valores negativos. Al ser una expansión del suelo, mientras su valor sea más negativo (p.ej. - 0,09) se entenderá como una mayor recuperación del suelo posterior a los eventos de estrés. En comparación a la resistencia (COLE c) que son valores positivos y los valores más altos $(>0,09)$ indican una baja capacidad de resistir el efecto del estrés.

En términos estadísticos, se utilizó estadística no paramétrica para cada uno de los parámetros evaluados. Estos se presentan en diagramas de caja (McGill et al., 1978) que incluyen la prueba de Kruskal-Wallis, que entrega las diferencias estadísticamente significativas en función de la mediana. Para evaluar la relación entre el carbono orgánico con la resistencia y resiliencia, se realizó una matriz de correlación lineal Spearman.

\section{RESULTADOS Y DISCUSIÓN}

\section{Descripción de las características generales del suelo}

De la caracterización general del suelo (Cuadro 1, Figura 2), se desprende información valiosa para comprender la unidad de suelo estudiada. El cambio más drástico y evidente se produce con el cambio de uso del suelo, ya que provoca la exposición superficial del 
Cuadro 1. Propiedades físico-químicas utilizadas como herramienta de caracterización general del suelo.

Table 1. Physicochemical properties used as a tool for general characterization of the soil.

\begin{tabular}{|c|c|c|c|c|c|}
\hline $\begin{array}{l}\text { Uso de suelo y } \\
\text { profundidad }\end{array}$ & \multirow{2}{*}{$\begin{array}{l}\text { Clase } \\
\text { textural }\end{array}$} & $\rho_{s}$ & $\rho_{a}$ & \multirow{2}{*}{$\frac{\mathrm{CO}}{\ldots\left[\mathrm{g} \mathrm{kg}^{-1}\right] \ldots}$} & \multirow{2}{*}{$\begin{array}{c}\mathrm{Al}_{\text {ext. }} \\
\ldots\left[\mathrm{mg} \mathrm{kg}^{-1}\right] \ldots\end{array}$} \\
\hline$[\mathrm{cm}]$ & & \multicolumn{2}{|c|}{$\ldots \ldots \ldots\left[\mathrm{Mg} \mathrm{m}^{-3}\right] \ldots \ldots \ldots$} & & \\
\hline BN 5 & Franca & $1,08 \pm 0,05$ & $0,17 \pm 0,007$ & $289,4 \pm 2,7$ & $552,6 \pm 32,5$ \\
\hline BN 15 & Franca & $1,07 \pm 0,02$ & $0,40 \pm 0,006$ & $281,2 \pm 2,7$ & $1056,6 \pm 66,4$ \\
\hline PN 5 & Franca & $1,39 \pm 0,02$ & $0,25 \pm 0,004$ & $243,2 \pm 2,7$ & $3176,6 \pm 101,7$ \\
\hline PN 15 & Arcillosa & $1,78 \pm 0,02$ & $0,39 \pm 0,016$ & $166,7 \pm 5,2$ & $4780,0 \pm 318,4$ \\
\hline
\end{tabular}

$\rho_{s}$ : densidad de partículas; $\rho_{a}$ : densidad aparente; CO: carbono orgánico y $\mathrm{Al}_{\text {ext }}$ : aluminio extractable. Se presentan valores promedio \pm 1 error estándar.

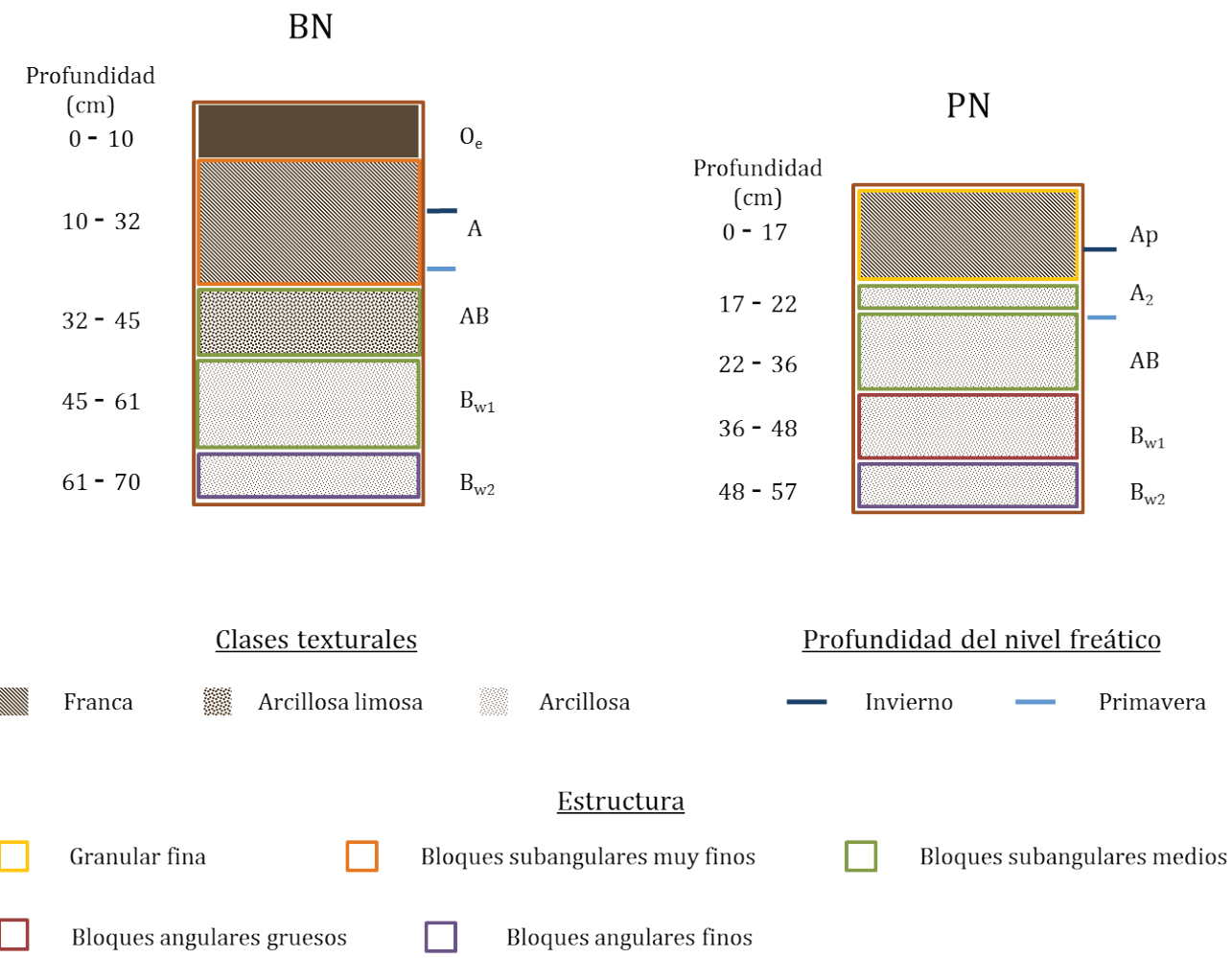

Figura 2. Esquema de los perfiles de suelo bajo el bosque nativo (BN) y pradera naturalizada (PN).

Figure 2. Soil profiles under native forest (NF) and naturalized grassland (NG).

horizonte A, reduciendo la profundidad total y efectiva del suelo, asociada a una discontinuidad textural $(57 \mathrm{~cm})$ bajo la condición pratense (Figura 2). De acuerdo al SAG (2001), la profundidad del suelo estudiado bajo bosque nativo (BN) es moderadamente profundo $(70 \mathrm{a} 90 \mathrm{~cm})$ y bajo pradera naturalizada (PN) es ligeramente profundo $(40 \mathrm{a} 70 \mathrm{~cm})$, pero se registraron sectores en donde el suelo es delgado (20 a $40 \mathrm{~cm})$.

Según CIREN (2003), la clase textural de la serie Alerce varía de franca $(0-18 \mathrm{~cm})$ a franco arcillo limosa $(18-32 \mathrm{~cm})$ en profundidad. En general, lo revisado in situ coincide con lo expuesto anteriormente, encon- trándose diferencias sólo para PN a $15 \mathrm{~cm}$ ya que se determinó que su clase textural corresponde a arcillosa (Figura 2). El contenido de aluminio extractable $\left(\mathrm{Al}_{\text {ext }}\right)$ es un parámetro utilizado en la clasificación de suelos (Soil Survey Staff, 2006), ya que, refleja el tipo de arcilla presente (Bonomelli et al., 2003). Según Marabolí (2008), la reactividad de las arcillas con respecto a la adsorción específica y no específica, en los suelos del sur de Chile, depende del contenido y tipo de arcilla. Esta reactividad tiene relación directa con los valores de $\mathrm{Al}_{\text {ext }}$. Los suelos Trumaos y Ñadis habilitados, presentan niveles superiores a $1.000 \mathrm{mg} \mathrm{kg}^{-1} \mathrm{de} \mathrm{Al}_{\text {ext' }}$ lo que 
implica un predominio de arcillas alofánicas. Aunque el suelo bajo $\mathrm{BN}$ a $5 \mathrm{~cm}$ presentó sólo $552,60 \pm 32,54$ $\mathrm{mg} \mathrm{kg}^{-1} \mathrm{de} \mathrm{Al}_{\text {ext' }}$ lo anterior no debería ser considerado definitivo para clasificar la reactividad de este tipo de suelo, ya que como se observa en la Figura 2, el BN presenta un horizonte orgánico $\left(\mathrm{O}_{\mathrm{e}}\right)$ en los primero 10 $\mathrm{cm}$, el cual no presenta la PN. En ese sentido, el suelo presentó altos valores de carbono orgánico (CO), bajo ambos usos, siendo mayor en superficie que en profundidad. En BN se encontraron los valores más altos de CO $(28,9 \%$ y $28,1 \%$, a 5 y $15 \mathrm{~cm}$, respectivamente) que son coincidentes con lo descrito por CIREN (2003).

El cambio de uso de suelo es la primera causa de modificaciones que ocurren en las características dinámicas del suelo, esto afecta a los sistemas biológicos, pero es una de las opciones para soportar y satisfacer las crecientes demandas humanas (Foley et al., 2005). Dentro de las características dinámicas que se modifican por el uso del suelo se encuentra la densidad aparente $\left(\rho_{a}\right)$, en donde FAO (2006) señala que los suelos formados a partir de materiales volcánicos recientes poseen una $\rho_{a} \leq 0,9 \mathrm{Mg} \mathrm{m}^{-3}$. Los valores de densidad aparente presentaron variaciones entre 0,17 $\pm 0,007$ (BN) y $0,25 \pm 0,004 \mathrm{Mg} \mathrm{m}^{-3}$ (PN) en los primeros $5 \mathrm{~cm}$, para luego aumentar en profundidad, lo que coincide con lo descrito por CIREN (2003). Ellies et al. (2000), sugieren que las diferencias presentadas entre los usos al que está sometido el suelo, se deben a cambios en su estructura, producto de los efectos mecánicos asociados al pisoteo animal en PN, lo que genera una reducción en la porosidad secundaria ( $>50 \mu \mathrm{m}$ de $\emptyset$ ) y un aumento en el asentamiento del suelo. Sin embargo, en este estudio no se puede descartar que los bajos valores de $\rho_{a}$ en $\mathrm{BN}$, en parte se deban a que la profundidad de recolección de las muestras tomó parte del horizonte Oe (Figura 2), el que se perdió con el cambio de uso de suelo en la pradera.

\section{Efecto del historial de manejo del suelo y de los eventos de estrés hidráulico y mecánico sobre el funcionamiento de los poros del suelo}

La capacidad de aire (CA) del suelo (Figura 3a - 3b) aumentó con el efecto del estrés hidráulico (EH), en ambos usos de suelos y profundidades, lo que concuerda con lo concluido por Seguel y Horn (2006); Peng et al. (2007); Ivelic-Sáez et al. (2015). Existen reportes que aseguran que los Andisoles poseen una alta capacidad de contracción, cuando disminuye su contenido de agua (Dörner et al, 2009; 2013). Por lo tanto, los procesos

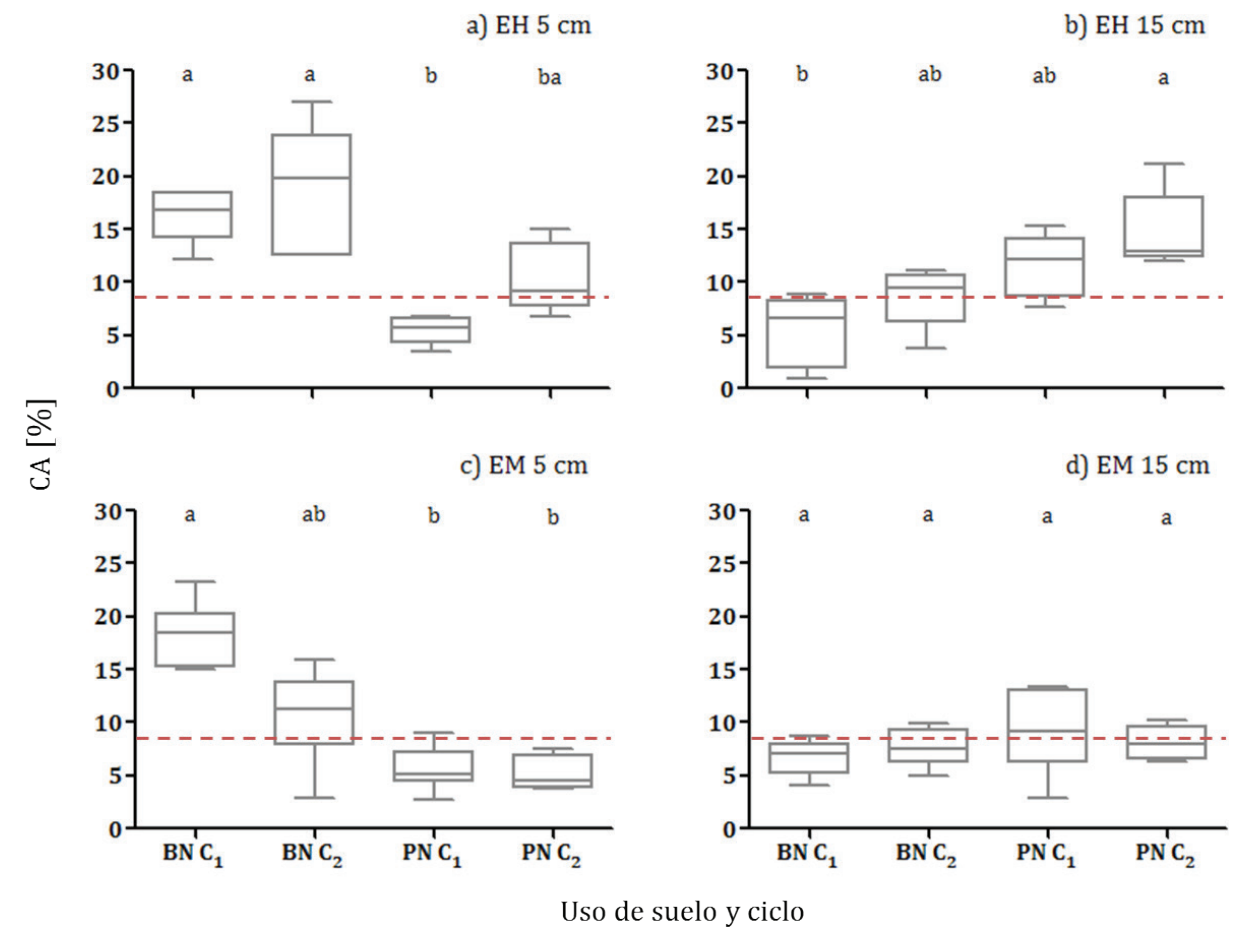

Figura 3. Capacidad de aire (CA) inicial $\left(C_{1}\right)$ y posterior $\left(C_{2}\right)$ a eventos de estrés hidráulico $(a-b)$ y mecánico $(c-d)$ a distintas profundidades ( 5 y $15 \mathrm{~cm}$ ) para ambos usos de suelo (BN: bosque nativo y PN: pradera naturalizada). Línea roja corresponde al valor crítico para la CA, que indica problemas de aireación por compactación (Horn y Fleige, 2009).

Figure 3. Air capacity $(C A)$ before $\left(C_{1}\right)$ and after $\left(C_{2}\right)$ the hydraulic $(a-b)$ and mechanical $(c-d)$ stresses at different soil depths ( 5 and $15 \mathrm{~cm}$ ) for both soil uses (BN: native forest and PN: naturalized grassland). Red line indicates the critical value for AC, which indicates aeration problems due to compaction (Horn and Fleige, 2009). 
de secado y re-humectación, que difieren según el uso del suelo (Dörner et al., 2009), son un mecanismo que contribuyen a la formación de estructura, y en este caso a la creación de poros que se manifiesta en un incremento en la CA. Es así que los suelos bajo $\mathrm{PN}$ a $5 \mathrm{~cm}\left(\mathrm{C}_{1}\right)$ y el $\mathrm{BN}$ a $15 \mathrm{~cm}\left(\mathrm{C}_{1}\right)$ de profundidad (Figura $3 \mathrm{a}$ y $3 \mathrm{~b}$ ), presentaron valores de CA bajo el umbral crítico del $8 \%$ (5,8\% y $6,7 \%$ respectivamente), los que indican problemas de aireación por compactación en suelos bajo cultivo (Horn y Fleige, 2009). Sin embargo, luego del EH $\left(\mathrm{C}_{2}\right)$ la CA alcanzó niveles que superaron dicho valor crítico $(9,1 \%$ y $9,5 \%$, respectivamente). Fisher y Binkley (2000) mencionan que en suelos forestales, cuando la densidad aparente sea $\geq \mathrm{a} 1,25 \mathrm{Mg} \mathrm{m}^{-3}$ y la macroporosidad $\leq$ a $20 \%$, se producen condiciones desfavorable para el desarrollo radical (mayor compactación, mayor resistencia a la penetración de raíces y mayores condiciones anaeróbicas). Estos valores no son completamente comparables con los del presente estudio ya que los valores de densidad aparente en los Andisoles son más bajos, encontrándose fuera de los rangos más comunes para los suelos en general (ver Cuadro 1).

En cuanto al efecto del estrés mecánico (pisoteo animal), se observó que en la mayoría de los casos, excepto en BN a $15 \mathrm{~cm}$ la CA disminuyó (Figura 3d). El efecto del estrés mecánico (EM) en la disminución de la macroporosidad (CA) ha sido reportado por Dörner et al. (2011) en Andisoles de Chile ellos concluyen que mientras el EH es capaz de favorecer la formación de estructura del suelo, el EM la destruye, disminuyendo así la CA. Dörner et al. (2009), mencionan que la presión mecánica no puede cambiar los poros de texturales (mesoporos ó poros no rígidos), pero puede compactar los poros estructurales (macroporos ó poros rígidos), especialmente los macroporos. En ese contexto, se sostiene que el cambio de uso de suelo (CUS) (v.g. desde un bosque nativo a una pradera) genera pérdidas en la porosidad total del suelo, existiendo una redistribución en la frecuencia del tamaño de los poros (Ferreras et al., 2007), lo que es relevante ya que el sistema poroso es el medio que permite el anclaje de las raíces de las plantas (Sheffer y Schachtschabel, 2010).

Ellies (1995) asegura que este aumento de la CA, se asocia a una reducción de los poros medios (10 - 0,2 $\mu \mathrm{m}$ de $\emptyset$ ) los cuales permiten retener agua útil para las plantas. Dörner et al. (2009; 2013) señalan, que la formación de meniscos de agua durante el proceso de secado, provoca una contracción del suelo, lo que trae como consecuencia un cambio en la distribución del volumen de poros. En la Figura 4a, se observó que

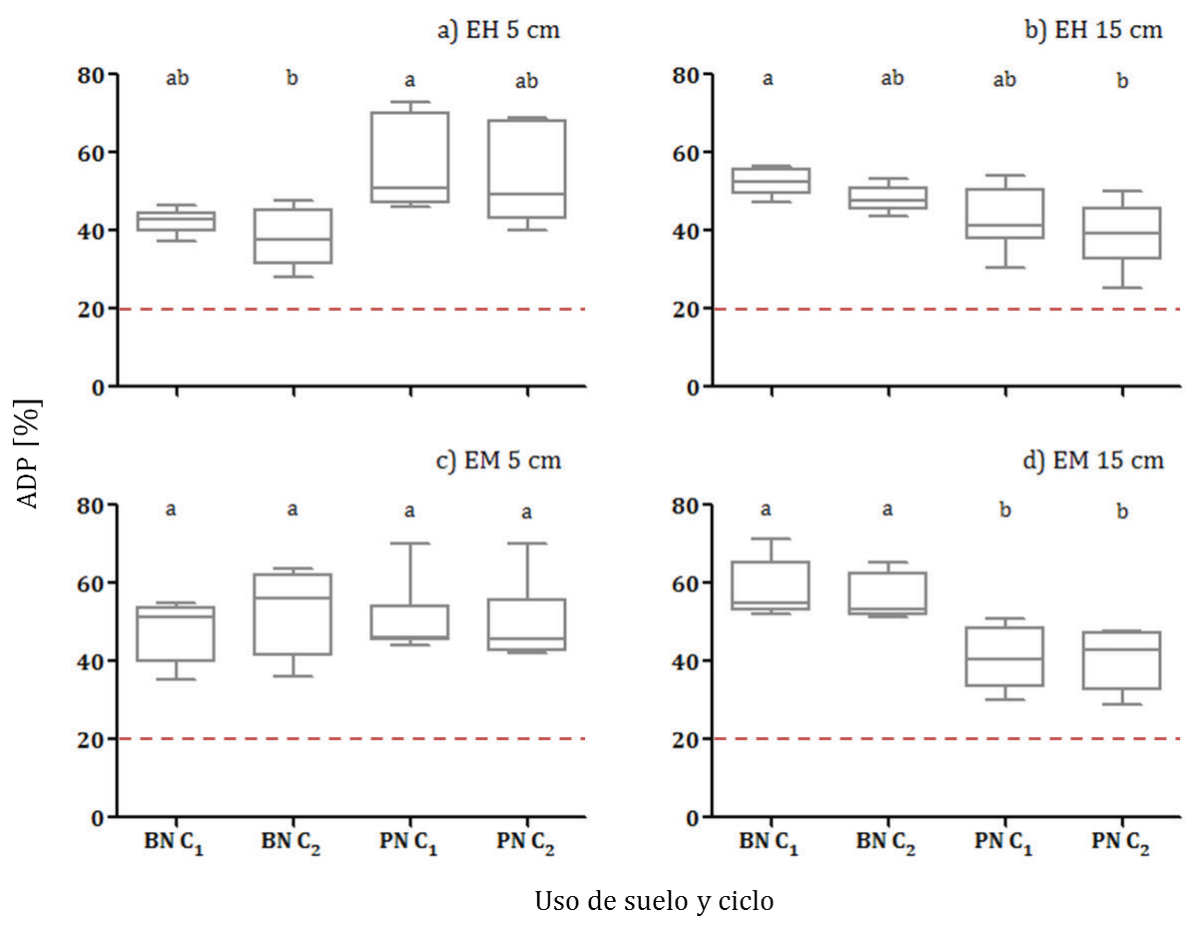

Figura 4. Contenido de agua disponible para las plantas (ADP) inicial $\left(C_{1}\right)$ y posterior $\left(C_{2}\right)$ a eventos de estrés hidráulico (a - b) y mecánico $(\mathrm{c}-\mathrm{d})$ a distintas profundidades $(5$ y $15 \mathrm{~cm}$ ) para ambos usos de suelo (BN: bosque nativo y PN: pradera naturalizada). Línea roja corresponde al valor óptimo de ADP para la producción vegetal (Reynolds et al., 2009).

Figure 4. Plant available water content $(A D P)$ before $\left(C_{1}\right)$ and after $\left(C_{2}\right)$ the hydraulic $(a-b)$ and mechanical $(c-d)$ stress at different depths (5 and $15 \mathrm{~cm}$ ) for both land uses (BN: native forest and PN: naturalized grassland). Red line indicates the optimum value of PAWC for crop production (Reynolds et al., 2009). 
el ADP (5 cm) era superior en PN $(51,1 \%)$ que en BN $(42,7 \%)$ en la primera curva de retención de agua $\left(\mathrm{C}_{1}\right)$. En ese contexto, Ellies (1995) menciona que un uso más intensivo del suelo (PN) disminuye la capacidad de aire del suelo y favorece la capacidad de retención de agua disponible para las plantas. Posterior al EH $\left(\mathrm{C}_{2}\right)$ se observó que en ambos usos de suelo y profundidades la mediana disminuyó, pero no hubo diferencias estadísticamente significativas $(p>0,05)$ entre $C_{1}$ y $C_{2}$. Además, el parámetro de ADP en ambos usos de suelo y profundidades se mantuvo sobre el 20\%, considerado "ideal", para la producción vegetal (Reynolds et al., 2009).

Los eventos de compactación no sólo disminuyen la CA, ya que la literatura señala que, esta pérdida de la estructuración permite que la porosidad media (10 - 2 $\mu \mathrm{m}$ de $\emptyset$ ) tenga una mayor participación en la porosidad total del suelo, favoreciendo la capacidad de retención de agua disponible para las plantas (Ellies et al., 2000; Dörner et al., 2009). Los resultados obtenidos, para evaluar el efecto del EM (Figura 4c - 4d) muestran un aumento $\left(\mathrm{C}_{2}\right)$ del ADP sólo en $\mathrm{BN}$ a $5 \mathrm{~cm}(4,5 \%)$ y PN a $15 \mathrm{~cm}(2,5 \%)$ de profundidad. La PN a $5 \mathrm{~cm}$ tuvo una variación levemente negativa $(0,3 \%)$, lo que refleja que el suelo ha estado sometido históricamente a niveles similares de estrés al aplicado en laboratorio, lo que es consistente con lo discutido por Baumgartl y Köck (2004).

El aumento en la intensidad del uso del suelo produjo una disminución en la conductividad de aire: $k_{l}$ en BN a $5 \mathrm{~cm}>k_{l}$ PN a $5 \mathrm{~cm}$ (Figura $5 \mathrm{a}-5 \mathrm{~b}$ ). Lo anterior es ratificado por estudios previos conducidos por Dörner et al. (2009) e Ivelic-Sáez et al. (2015). Como se mencionó, el EM fue evaluado en función a la presión ejercida por la pezuña ovina. Ésta, causa una deformación superficial del suelo y ocurre un reordenamiento del espacio poroso, lo que conduce a un aumento de la resistencia a la penetración y una reducción de la macroporosidad (Dörner et al, 2009). La porosidad gruesa es responsable del transporte de aire y cuando el suelo es sometido a eventos de EM, son los primeros en reducirse (Richard et al., 2001), ya que se deforman con facilidad (Gebhardt et al., 2009). Por lo tanto, el EM disminuye la conductividad del aire en el suelo (Dörner et al, 2009). Este efecto fue visible en ambos usos y profundidades de suelo (Figura $5 c-5 d$ ), siendo más notorio en el BN ya que tuvo un mayor contenido de macroporos (CA). a) $\mathrm{EH} 5 \mathrm{~cm}$

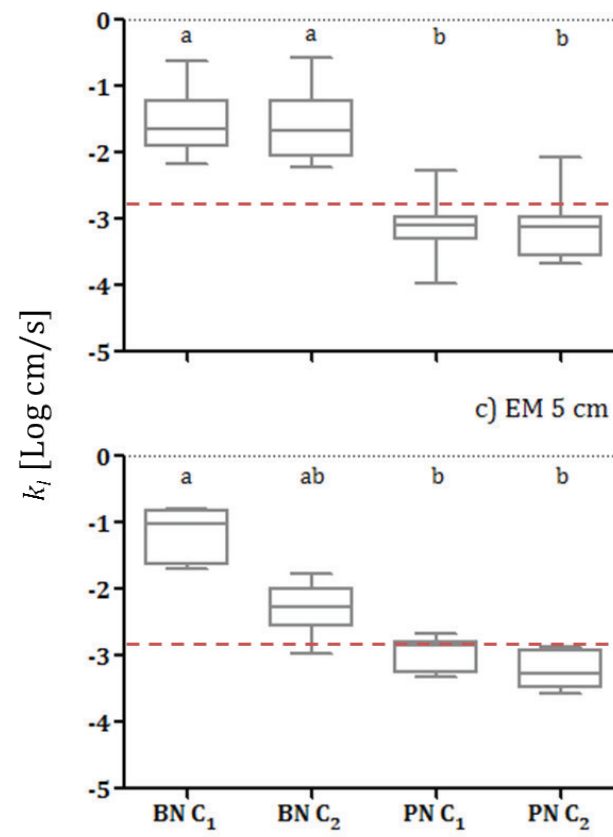

b) $\mathrm{EH} 15 \mathrm{~cm}$

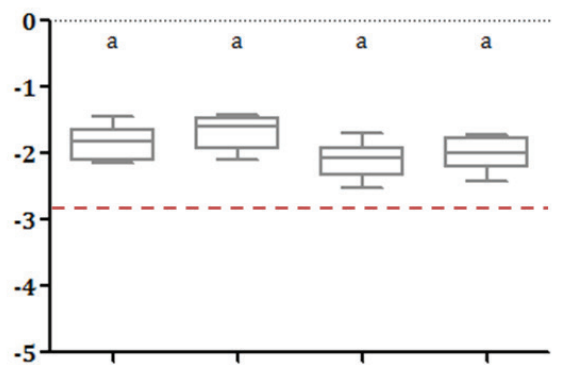

d) $\mathrm{EM} 15 \mathrm{~cm}$

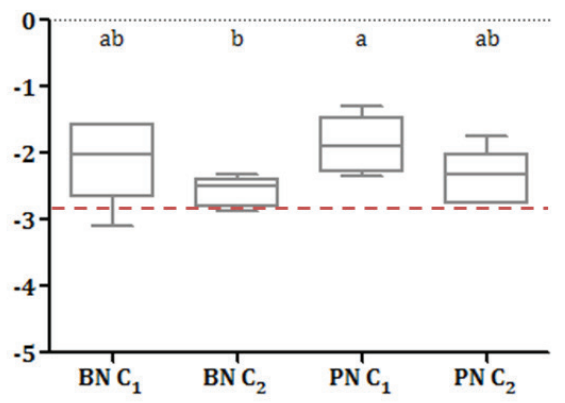

Uso de suelo y ciclo

Figura 5. Conductividad de aire $\left(k_{l}\right)$ inicial $\left(C_{1}\right)$ y posterior $\left(C_{2}\right)$ a eventos de estrés hidráulico $(a-b)$ y mecánico $(c-d)$ a distintas profundidades (5 y $15 \mathrm{~cm}$ ) para ambos usos de suelo (BN: bosque nativo y PN: pradera naturalizada). Línea roja corresponde al valor crítico de $k_{p}$ que indica problemas de aireación por compactación (Horn y Fleige, 2009).

Figure 5. Air conductivity $\left(k_{l}\right)$ before $\left(C_{1}\right)$ and after $\left(C_{2}\right)$ the hydraulic $(a-b)$ and mechanical $(c-d)$ stresses at different soil depths ( 5 and $15 \mathrm{~cm}$ ) for both land uses (BN: native forest and PN: naturalized grassland). Red line indicates the critical value of $k_{p}$, indicating problems aeration due to compaction (Horn and Fleige, 2009). 


\section{Índice de resistencia y resiliencia de los poros del suelo}

El suelo presentó una mayor resistencia al efecto del EH que el EM (Figura 6a - 6b). Esto, debido a que para el $\mathrm{EH}$ en ambos usos de suelos y profundidades presentaron un índice de contracción que varió de muy bajo a moderado, en comparación al EM que varió de alto a muy alto, según lo descrito por Peng et al. (2007). Al evaluar la recuperación del volumen del suelo, representado por la resiliencia (COLE r), los valores tienden a ser negativos porque es un recuperación en contra de la fuerza de gravedad (mientras más negativo sea el valor COLE r mayor es la recuperación del suelo). Aquí los efectos fueron distintos según el tipo de estrés aplicado (Figura 6c-6d). Para ambos usos de suelos y profundidades, las muestras sometidas al EM presentaron una mayor capacidad de recuperación en comparación a las sometidas al EH; sin embargo, hay que considerar que el EM fue el que generó la mayor deformación de las muestras. Además, el BN como uso de suelo presentó la mayor resiliencia (COLE r presentó valores más negativos) en ambos tipos de estrés.
Por otra parte, la amplia distribución de los datos del COLE (para ambos usos y profundidades) dificulta la percepción del daño y la recuperación de las muestra, y aunque el suelo Ñadi bajo BN a 5 y $15 \mathrm{~cm}$ presentó medianas de una moderada capacidad de recuperación (COLE c para el EM), no se puede concluir que el equilibrio hídrico del sistema poroso sometido al EM no se verá afectado a futuro.

El suelo estudiado fue susceptible a la deformación ya sea por acción de fuerzas internas o externas. La contracción de los Andisoles está estrechamente influenciada por los altos contenidos de CO, la presencia de alofán y su tipo de estructura (Dörner et al., 2013). Las fuerzas externas conducen a la deformación del suelo (COLE EM), especialmente cuando el uso es menos intensivo como en el caso de suelos bajo bosque (Dörner et al. 2009). A su vez, fuerzas internas (ciclos de humectación y secado) también favorecen la deformación del suelo (COLE EH), pero, el efecto del estrés hidráulico sobre índice de contracción es generalmente menor a la deformación generada por el EM. Lo anterior es mencionado por Dörner et al. (2011), quienes señalan que existe una relación de 1:1 entre el COLE EM
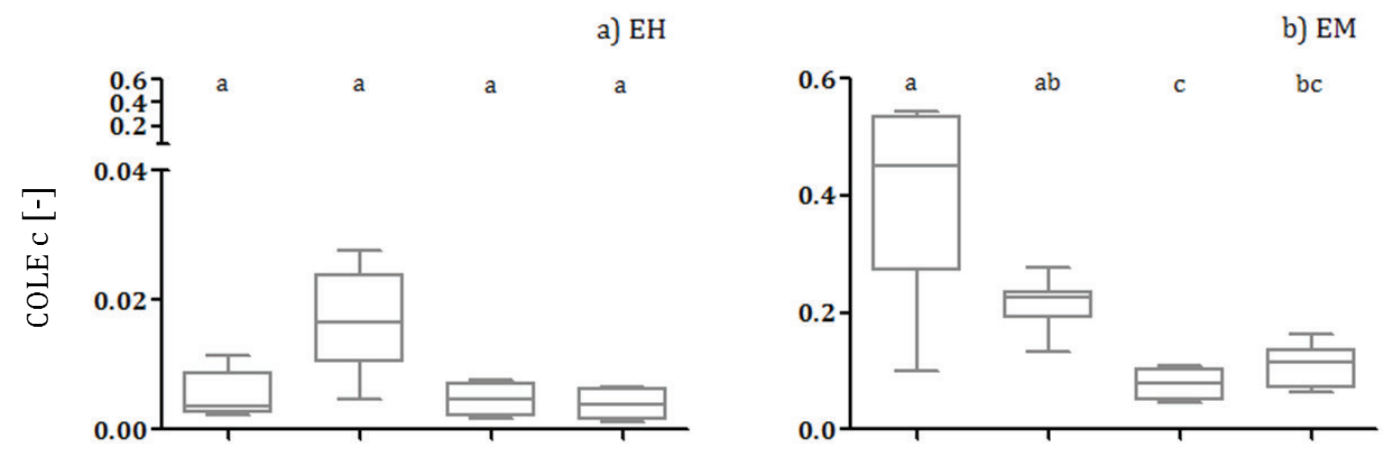

c) $\mathrm{EH}$
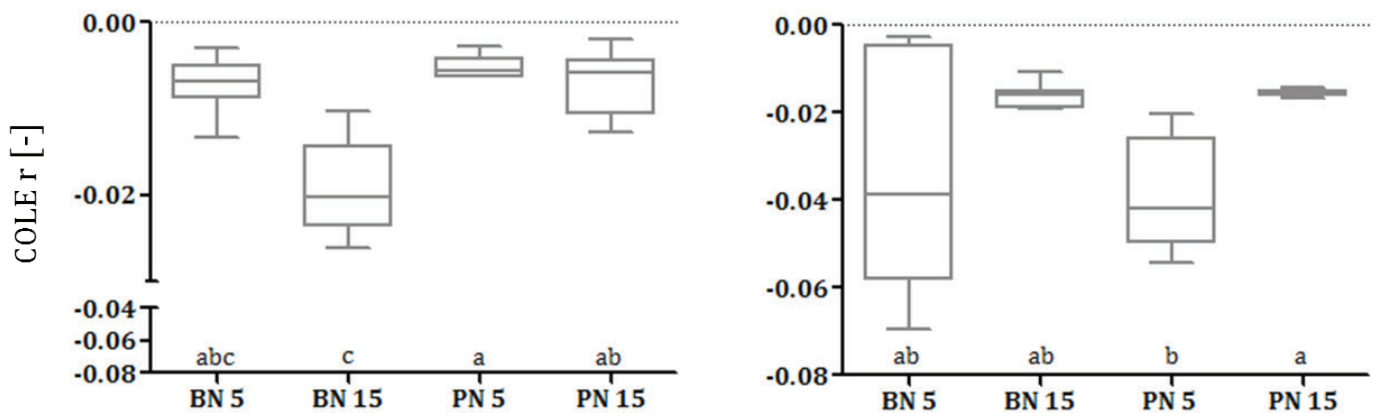

Uso de suelo y profundidad $[\mathrm{cm}]$

Figura 6. Coeficiente de extensibilidad lineal para la resistencia (COLE c) y resiliencia (COLE r), luego de ser sometido a eventos de estrés hidráulico (a - c) y mecánico (b - d) a distintas profundidades (5 y $15 \mathrm{~cm})$ para ambos usos de suelo $(\mathrm{BN}$ : bosque nativo y PN: pradera naturalizada).

Figure 6. Coefficient of linear extensibility for resistance (COLE c) and resilience (COLE r), after undergoing a hydraulic (a - c) and mechanical $(\mathrm{b}-\mathrm{d})$ stress at different depths $(5-15 \mathrm{~cm})$ for both land uses (BN: native forest and PN: naturalized grassland). 
y COLE EH, pero la contracción generada por el EM es superior al EH, resaltando que la magnitud de la deformación por formación de meniscos es menor a la causada por agentes externos (Dörner et al., 2009). Seguel y Horn (2006) aseguran que el suelo está condicionado a modificaciones de su volumen por la ocurrencia de ciclos naturales de mojado y secado, pero a estas, se les suman las que dependen directamente del manejo del suelo. Lo anterior, evidencia que el suelo no se comporta como un cuerpo rígido, sino como uno dinámico, que está sujeto a variaciones en la matriz porosa debido a eventos naturales y también antropogénicos.

\section{Relación entre la resistencia y resiliencia del suelo con su contenido de materia orgánica}

Murray et al. (2011), indican que los cambios en el uso del suelo conllevan a una modificación de la composición vegetal. También, argumentan que los cambios en el uso afectan el contenido y tipo de la materia orgánica de los suelos, los cuales modifican las propiedades físicas como la estructura y la densidad aparente, lo cual concuerda con lo presentado en el Cuadro 1, así como la porosidad total.

En el presente trabajo que el carbono orgánico se correlaciona de mejor manera con la resistencia (COLE c EM: 0,76 $p>0,05$ - 12) y la resiliencia (COLE c EM: $0,71-p>0,05-12$ ) mecánica del suelo (Cuadro 2). Gregory et al. (2009) señalan que la resiliencia y resistencia del suelo están estrechamente relacionadas con la materia orgánica presente en el suelo. De esta forma, Dörner et al. (2011) mencionan que el efecto de la materia orgánica no es tan visible para el EH, pero que está altamente correlacionada con el comportamiento mecánico del suelo. En esa misma línea, Ellies (1988) señala que los componentes orgánicos incrementan la elasticidad de los suelos derivados de cenizas volcánicas, lo que al mismo tiempo esta estrechamente relacionado con la resiliencia funcional de los poros (Dörner et al., 2011).

\section{CONCLUSIONES}

El cambio de uso de suelo, desde un bosque nativo $(\mathrm{BN})$ a una pradera naturalizada (PN), provocó un cambio morfológico importante en el perfil de suelo, que se traduce en una reducción de la profundidad y en la exposición del horizonte A en la superficie de PN. Por otro lado, las características funcionales de los poros están condicionadas por el uso actual del suelo Ñadi. Es así que, mientras el BN presentó una alta capacidad de almacenar y conducir de aire (CA y $k_{l}$ ) en desmedro del

Cuadro 2. Matriz de correlación lineal.

Table 2. Linear correlation matrix.

\begin{tabular}{|c|c|c|c|c|c|c|}
\hline Variable & $\mathrm{CO}$ & COEL C & COEL $r$ & COEL C & COEL $r$ & Valores \\
\hline & & \multicolumn{2}{|c|}{ EH } & \multicolumn{2}{|c|}{ EM } & \\
\hline & & 0,59 & 0,61 & 0,76 & 0,71 & Rs \\
\hline \multirow[t]{3}{*}{$\mathrm{CO}$} & 1,00 & 0,041 & 0,037 & 0,004 & 0,009 & $p$ \\
\hline & & 12 & 12 & 12 & 12 & $\mathrm{n}$ \\
\hline & & & 0,44 & 0,29 & 0,57 & Rs \\
\hline \multirow[t]{3}{*}{ COEL c EH } & & 1,00 & 0,032 & 0,167 & 0,003 & $p$ \\
\hline & & & 24 & 24 & 24 & $\mathrm{n}$ \\
\hline & & & & 0,41 & 0,57 & Rs \\
\hline \multirow[t]{3}{*}{ COEL r EH } & & & 1,00 & 0,047 & 0,003 & $p$ \\
\hline & & & & 24 & 24 & $\mathrm{n}$ \\
\hline & & & & & 0,69 & Rs \\
\hline \multirow[t]{3}{*}{ COEL c EM } & & & & 1,00 & 0,000 & $p$ \\
\hline & & & & & 27 & $\mathrm{n}$ \\
\hline & & & & & & Rs \\
\hline \multirow[t]{2}{*}{ COEL r EM } & & & & & 1,00 & $p$ \\
\hline & & & & & & $\mathrm{n}$ \\
\hline
\end{tabular}

Los valores de correlación (Rs) en negrita fueron significativos a $p<0,05$. 
volumen de agua disponible para las plantas (ADP), la pradera naturaliza (PN) se caracterizó por un alto volumen de ADP, pero menor CA y $k_{r}$.

El efecto del estrés hidráulico (EH) sobre el funcionamiento de los poros se caracterizó por i) inducir un aumento de la capacidad y conducción de aire y ii) provocar una disminución del agua disponible para las plantas. Por otro lado, el estrés mecánico (EM) tuvo un efecto negativo (disminución) en los parámetros de capacidad y conducción de aire (CA y $k_{l}$ ), y un efecto positivo (aumento) de volumen de poros que almacenan agua (ADP).

El efecto del estrés mecánico (EM) fue mayor que el estrés hidráulico (EH) en ambos usos de suelo y profundidades analizadas. La pradera naturalizada (PN) presentó una resistencia mayor a eventos de estrés mecánico e hidráulico. Sin embargo, el bosque nativo (BN) sufrió la mayor deformación en comparación al suelo bajo PN, pero se caracterizó por presentar una alta capacidad de recuperación independiente del tipo de estrés. En ese contexto, la resistencia y resiliencia funcional de los poros del suelo se correlaciona de forma positiva con el contenido de carbono orgánico (CO) que presentaron ambos usos de suelo.

\section{AGRADECIMIENTOS}

Este trabajo fue financiado por el Proyecto FONDECYT 1130546. Los autores agradecen al dueño del Predio Chaqueihua, Don Alfredo Núñez y Sra. Elba, por su excelente disposición para llevar a cabo ensayos en el sitio de estudio. Además, la primera autora agradece la ayuda entregada por todos los integrantes del grupo de investigación. El Dr. José Dörner agradece a la Fundación Alexander von Humboldt por otorgar la beca "Georg Forster Fellowship for Experienced Researchers" con la que fue posible una estadía de investigación en la Universidad de Kiel.

\section{REFERENCIAS}

Aguayo, M., Pauchard, A., Azócar, G., Parra, O., 2009. Cambio del uso del suelo en el centro sur de Chile a fines del siglo XX. Entendiendo la dinámica espacial y temporal del paisaje. Revista Chilena de Historia Natural 82(3), 361-374. http://www.scielo.cl/scielo.php?pid=S0716078X2009000300004\&script=sci_arttext

Baumgartl, Th., Köck, B., 2004. Modelling volume change and mechanical properties with hydraulic models. Soil Science Society of America Journal 68, 57-65. doi:10.2136/ sssaj2004.5700

Bonomelli, C., Henriquez, C., Giral, L., Bescansa, P., 2003. Disponibilidad de fósforo en un andisol, con distintas fuentes y dosis de fósforo, en condiciones controladas. Ciencia e Investigación Agraria 30(3), 186-192. https:// repositorio.uc.cl/handle/11534/8608

Centro de Información de Recursos Naturales (CIREN), 2003.
Estudio agrológico X Región. Descripción de Suelos, Materiales y Símbolos, Publicación CIREN Nº 123, Santiago, Chile.

Dörner, J., Dec, D., Peng, X., Horn, R., 2009. Efecto del cambio de uso en la estabilidad de la estructura y la función de los poros de un Andisol (Typic Hapludand) del sur de Chile. Journal of Soil Science and Plant Nutrition 9(3), 190-209. http://www.scielo.cl/scielo.php?pid=S071827912009000300003\&script=sci_arttext

Dörner, J., Dec, D., Zúñiga, F., Sandoval, P., Horn, R., 2011. Effect of land use change on Andosol's pore functions and their functional resilience after mechanical and hydraulic stress. Soil and Tillage Research 115-116, 7179. http://www.sciencedirect.com/science/article/pii/ S0167198711001310

Dörner, J., Dec, D., Sáez, C., Peng, X., Ivelic-Sáez, J., Zúñiga, F., Seguel, O. Horn, R., 2013. The shrinkage properties of different managed andisols as function of aggregate scale. Agro Sur 41(1): 1-9. http://mingaonline.uach.cl/scielo. php?pid=S0304-88022013000100001\&script=sci_arttext

Ellies, A., 1988. Mechanical consolidation in volcanic ash soils, in: Drescher, J., Horn, R., De Boodt, M. (Eds.), Impact of Water and External Forces on Soil Structure, Catena Supplement 11. Catena Verlag, Cremlingen-Destedt, W. Germany, pp. 87-92.

Ellies, A., 1995. Efecto del manejo sobre las propiedades físicas de suelos trumaos y rojo arcillosos. Bosque 16(2), 101-110. http://mingaonline.uach.cl/pdf/bosque/ v16n2/art09.pdf

Ellies, A., Horn, R., Smith, R., 2000. Effect of management of a volcanic ash soil on structural properties. International Agrophysics 14, 377-384. http://www.ōld.international-agrophysics.org/en/issues.html?stan=detail\&vol=1 $4 \&$ numer $=4 \&$ paper $=249 \& \mathrm{i}=1$

Ferreras, L., Magra, G., Besson, P., Kovalevski, E., García, F., 2007. Indicadores de calidad física en suelos de la Región Pampeana Norte de Argentina bajo siembra directa. Ciencia del suelo 25(2), 159-172. http://www.scielo.org.ar/scielo.php?pid=S185020672007000200007\&script=sci_arttext

Fisher, R., Binkley, D., 2000. Ecology and management of forest soils, third ed. John Wiley \& Sons. West Sussex, UK.

Foley, J., Defries, R., Asner, G., Barford, C., Bonan, G., Carpenter, S., Chapin, S., Coe, M., Daily, G., Gibbs, H., Helkowski, J. Holloway, T., Howard, E., Kucharik, C., Monfreda, C., Patz, J. Prentice, C., Ramankutty, N., Snyder, P., 2005. Global Consequences of Land Use. Science 309, 570. http:// www.sciencemag.org/content/309/5734/570.full.html

Food and Agriculture Organization of the United Nations (FAO), 2006. Guidelines for Soil Description, Fourth ed. Rome, Italy. ftp://ftp.fao.org/agl/agll/docs/guidel_soil_ descr.pdf

Forsythe, W., 1985. Física de suelos. Manual de laboratorio. Instituto Interamericano de Cooperación para la Agricultura (IICA), San José, Costa Rica.

Gebhardt, S., Fleige, H., Horn, R., 2009. Effect of compaction on pore functions of soils in a Saalean moraine landscape in northern Germany. Journal of Plant Nutrition and Soil Science 172, 601-614. http://onlinelibrary.wiley. com/doi/10.1002/jpln.200800073/epdf 
Gregory, A., Watt, C., Griffiths, B., Hallet, P., Kuan, H., Whitmore, P., 2009. The effect of long-term soil management on the physical and biological resilience of a range of arable and grassland soil in England. Geoderma 153, 172-185. http://www.sciencedirect.com/science/article/pii/ S0016706109002444

Horn, R., Fleige, H., 2009. Risk assessment of subsoil compaction for arable soils in Northwest Germany at farm scale. Soil and Tillage Research 102, 201-208. http://www.sciencedirect.com/science/article/pii/ S0167198708001074

Horn, R., Kutilek, M., 2009. The intensity-capacity concept. How far is it posible to predict intensity values with capacity parameters. Soil and Tillage Research 103, 1-3. http://www.sciencedirect.com/science/article/pii/ S0167198708002055

Ivelic, J., 2013. Efecto de estrategias de mejoramiento de praderas sobre la resistencia y resiliencia funcional del sistema poroso de un Andisol bajo pastoreo ovino. Tesis Magíster Ciencias del suelo. Universidad Austral de Chile.

Ivelic-Sáez, J., Zúñiga, F., Valle, S., López, I., Dec, D., Dörner, J., 2015. Functional resistance and resilience of the pore system of an Andisol exposed to different strategies of pasture improvement under sheep grazing. Journal of Soil Science and Plant Nutrition 15(3), 663679. http://www.scielo.cl/scielo.php?pid=S071895162015000300010\&script=sci_arttext

Karlen, D., Mausbach, M., Doran, J., Cline, R., Harris, R., Schuman, G., 1997. Soil quality: a concept, definition and framework for evaluation. Soil Science Society of America Journal 61, 4-10. doi:10.2136/ sssaj1997.03615995006100010001x

Krümmelbein, J., Wang, Z., Zhao, Y., Peth, S., Horn, R., 2006. Influence of various grazing intensities on soil stability, soil structure and water balance of grassland soils in Inner Mongolia, P.R. China, in: Horn, R., Fleige, H., Peth, S., Peng, X. (Eds.), Management for sustainability. Advances in GeoEcology. Catena Verlag, Reiskirchen 38, pp. 93-101.

Luzio, W., 2010. Suelos de Chile. Departamento de Ingeniería y Suelos, Facultad de Ciencias Agronómicas, Universidad de Chile, Santiago.

Marabolí, A., 2008. Proposición de factores de equivalencia de concentraciones de nutrientes entre profundidades de muestreo en suelos volcánicos. Tesis Licenciado en Agronomía, Universidad Austral de Chile. 91 p.

Martínez, E., Fuentes, J.P., Acevedo, E., 2008. Carbono orgánico y propiedades del suelo. Journal of Soil Science and Plant Nutrition 8, 68-96. http:// www.scielo.cl/scielo.php?script=sci_arttext\&pid $=$ S0718-27912008000100006

McGill, R., Tukey, J., Larsen, W., 1978. Variations of box plots. Journal of the American Statistical Association 32, 1216. doi:10.1080/00031305.1978.10479236

Murray, R., Bojórquez, J., Hernández, A., Orozco, M., García, J., Gómez, R., Ontiveros, H., Aguirre, J., 2011. Efecto de la materia orgánica sobre las propiedades físicas del suelo en un sistema agroforestal de la llanura costera norte de Nayarit, México. Bio Ciencias 1(3), 27-35. http:// biociencias.uan.edu.mx/publicaciones/02-03/biociencias3-3.pdf

Peng, X., Horn, R., Smucker, A., 2007. Pore shrinkage dependency of inorganic and organic soils on wetting and drying cycles. Soil Science Society American Journal 71, 1095-1104. doi:10.2136/sssaj2006.0156

Reynolds, W., Drury, C., Tan, C., Fox. C., Yang, X., 2009. Use of indicators and pore volume-function characteristics to quantify soil physical quality. Geoderma 152, 252-263. http://www.sciencedirect.com/science/article/pii/ S0016706109001967

Richard, G., Cousin, I., Sillon, J., Bruand, A., Guerif, J., 2001. Effect of compaction on the porosity of a silty soil: influence on unsaturated hydraulic properties. European Journal of Soil Science 52, 49-58. http://onlinelibrary. wiley.com/doi/10.1046/j.1365-2389.2001.00357.x/ epdf

Sadzawka, A., Carrasco, M.A., Grez, R., Mora, M., Flores, H., Neaman, A., 2006. Métodos de análisis recomendados para los suelos de Chile. Revisión 2006. Instituto de Investigaciones Agropecuarias, Serie Actas INIA № 34, Santiago, Chile.

Sánchez, A., Morales, R., 2000. Regiones de Chile, tercera ed. Universitaria, Santiago, Chile.

Scheffer, F., Schachtschabel, P. 2010. Lehrbuch der Bodenkunde, 16. Auflage. Blume, H.-P., Brümmer, G.W., Horn, R., Kandeler, E., Kögel-Knabner, I., Kretzschmar, R., Stahr, K., Wilke, B.-M. (Eds.), Spektrum Akademischer Verlag, Heidelberg, Germany.

Schlatter, J., Grez, R., Gerding, V., 2003. Manual para el reconocimiento de suelos, tercera ed. Universidad Austral de Chile, Valdivia.

Seguel, O., Horn, R., 2006. Structure properties and pore dynamics in aggregate beds due to wetting-drying cycles. Journal of Plant Nutrition and Soil Science 169, 221232. http://onlinelibrary.wiley.com/doi/10.1002/ jpln.200521854/epdf

Servicio Agrícola y Ganadero (SAG), 2001. Pauta para Estudio de Suelos. http://historico.sag.gob.cl/common/ asp/pagAtachadorVisualizador.asp?argCryptedData $=\mathrm{G}$ P1TkTXdhRJAS2Wp3v88hHfRZcNgy4C1\&argModo=in line\&argOrigen=BD\&argFlagYaGrabados $=\& \operatorname{argArchivo}$ $\mathrm{Id}=14316$ (acceso, 07.05.2015).

Seybold, C., Herrick, J., Brejda, J., 1999. Soil resilience: a fundamental component of soil quality. Soil Science 164(4), 224-234.

Soil Survey Staff, 2006. Keys to Soil Taxonomy. $10^{\text {th }}$ ed. USDA, Natural Resources Conservation Service, Washington DC.

Thiers, O., Gerding, V., Lara, A., Echeverría, C., 2007. Variación de la napa freática en un suelo ñadi bajo diferentes tipos vegetacionales, X Región, Chile, in: Libro de actas de Eco Reuniones. Primera reunión sobre forestación en la patagonia EcoForestar 2007. Gonda, H., Davel, M., Loguercio, G., Picco, O. (Eds.), Centro de Investigación y Extensión Forestal Andino Patagónica (CIEFAP). Esquel, Chubut, Argentina, pp. 259-266. 